\title{
Sazonalidade e horário de atividade de abelhas Euglossinae (Hymenoptera, Apidae), em florestas de terra firme na Amazônia Central ${ }^{1}$
}

\author{
Marcio Luiz de Oliveira ${ }^{2,3}$
}

\begin{abstract}
Seasonality and daily activity of Euglossinae bees (Hymenoptera, Apidae) in terra firme forest in Central Amazonia. Euglossinae bee fauna of two areas of terra firme forest near Manaus, Amazonas, Brazil was compared. Over a twelve-month period collections were done fortnightly, between 7:00 a.m and 5:00 p.m, using traps with eight kinds of scent baits. The traps were placed in the understory and in tree crowns. The majority of species and specimens was most active between August-December, near the beginning of the rainy season in this region. The bees were most active from 9:00 a.m. until 4:00 p.m., while air temperatures were between $24.5-27^{\circ} \mathrm{C}$, but there was a decline in this activity when air temperature varied above or below of this range. There were differences in the seasonality and in the daily activity patterns for the two areas studied but these differences were not significative.

KEY WORDS. Orchid-bees, Euglossinae, daily activity, seasonality, Amazonia
\end{abstract}

Em trabalho realizado na Amazônia Central que visava verificar a existência de padrões de sazonalidade entre abelhas da sub-família Euglossinae, BECKER et al. (1991) encontraram resultados pouco conclusivos a esse respeito. Esses autores salientaram porém que seria interessante averiguar se a sazonalidade entre os Euglossinae estaria correlacionada com fenômenos, tais como a floração ou a precipitação.

Quanto ao horário de atividade dessas abelhas, DoDson et al. (1969) afirmam que os Euglossinae são mais ativos na parte da manhã e, por isso, realizaram seus experimentos no período de 7 às 13 horas. Baseados nessa informação, BRAGA (1976), Powell \& Powell (1987) e Becker et al. (1991), trabalhando na Amazônia Central, também realizaram seus experimentos em períodos semelhantes.

Como todos esses autores que estudaram Euglossinae na Amazônia Central, o fizeram em períodos muito curtos ou obtiveram resultados inconclusivos, os objetivos deste trabalho foram: verificar o período ao longo do ano e ao longo do dia em que as abelhas Euglossinae estão mais ativas, levando-se em conta as precipitações mensais e variações diárias da temperatura do ambiente, respectivamente, e verificar se os padrões encontrados são correspondentes em duas áreas de mata de terra firme.

1) Publicação número 192 do Projeto Dinâmica Biológica de Fragmentos Florestais (INPA-SI).

2) Departamento de Ciências da Natureza, Universidade Federal do Acre. Rodovia BR 364, Km 5, 69915-900 Rio Branco, Acre, Brasil.

3) Endereço para correspondência: Departamento de Biologia, Faculdade de Filosofia, Ciências e Letras de Ribeirão Preto, Universidade de São Paulo. 14040-901 Ribeirão Preto, São Paulo, Brasil.

E-mail: mlolivei@usp.br 


\section{MATERIAL E MÉTODOS}

A localização e caracterização das áreas de estudos, assim como o detalhamento da metodologia empregada, encontram-se em OliveIRA \& CAMPOS (1995).

A análise de correlação foi feita com os coeficientes de correlação paramétrico de Pearson (r) e não-paramétrico de Spearman (rs) para dados normais ou não, respectivamente. Os valores obtidos foram, então, submetidos ao teste " $t$ ".

\section{RESULTADOS}

A relação das 38 espécies coletadas ao longo dos meses se encontra na tabela I. A maioria das espécies e indivíduos foi mais comum no período compreendido entre agosto e dezembro (Figs 1,2), o que praticamente coincide com o fim da estação seca e início da estação chuvosa, sendo que esta ocorre, geralmente, de outubro a março nessa região (RIBEIRO \& ADIS 1984). Algumas espécies, além de muito raras, só foram capturadas nesta época, como foi o caso de Exaerete trochantherica (Friese, 1900); Exaerete smaragdina (Guérin, 1845); Eufriesea vidua (Moure, 1976); Euglossa analis Westwood, 1840; Euglossa bidentata Dressler, 1982; Euglossa prasina Dressler, 1982; Euglossa laevecincta Dressler, 1982; Euglossa sp. 1 e Euglossa sp. 3, ao passo que Euglossa piliventris Guérin, 1845 foi coletada exclusivamente na estação seca.

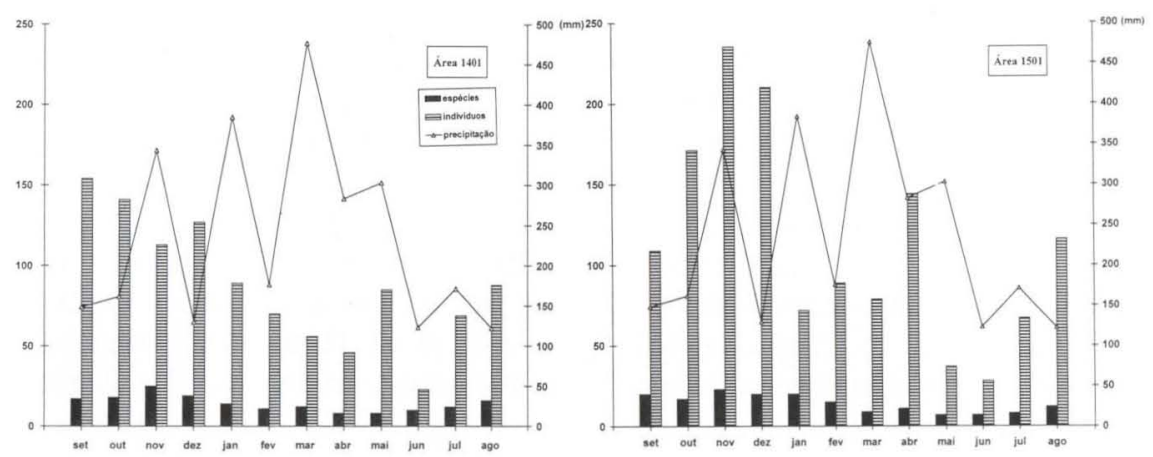

Figs 1-2. Comparação da sazonalidade de Euglossinae em duas áreas de mata contínua de terra firme na Amazônia Central, no período de setembro de 1989 a agosto de 1990 .

$\mathrm{Na}$ área 1401 houve um pico na abundância de indivíduos em dezembro e um pico menor em maio, sem que fossem acompanhados de respectivos picos no número de espécies, o que só aconteceu em novembro (Fig. 1). Diferentemente, a área 1501 apresentou picos na abundância de indivíduos em novembro, fevereiro e abril, sendo que desses, somente em fevereiro não houve um pico no número de espécies (Fig. 2). Apesar disso, quando as duas áreas foram comparadas, o padrão de sazonalidade para todas as espécies esteve correlacionado positivamente $(\mathrm{r}=$ $0,58 ; \mathrm{t}=2,26 ; \mathrm{p}<0,05 ; \mathrm{G} . \mathrm{L}=10)$. Houve correlação positiva também entre indivíduos e espécies ao longo do ano, na área 1401 ( $\mathrm{rs}=0,804 ; \mathrm{t}=4,31 ; \mathrm{p}<0,05$; $\mathrm{G} . \mathrm{L}=10)$ e na área $1501(\mathrm{rs}=0,744 ; \mathrm{t}=3,51 \mathrm{p}<0,05 ; \mathrm{G} . \mathrm{L} .=10)$. 


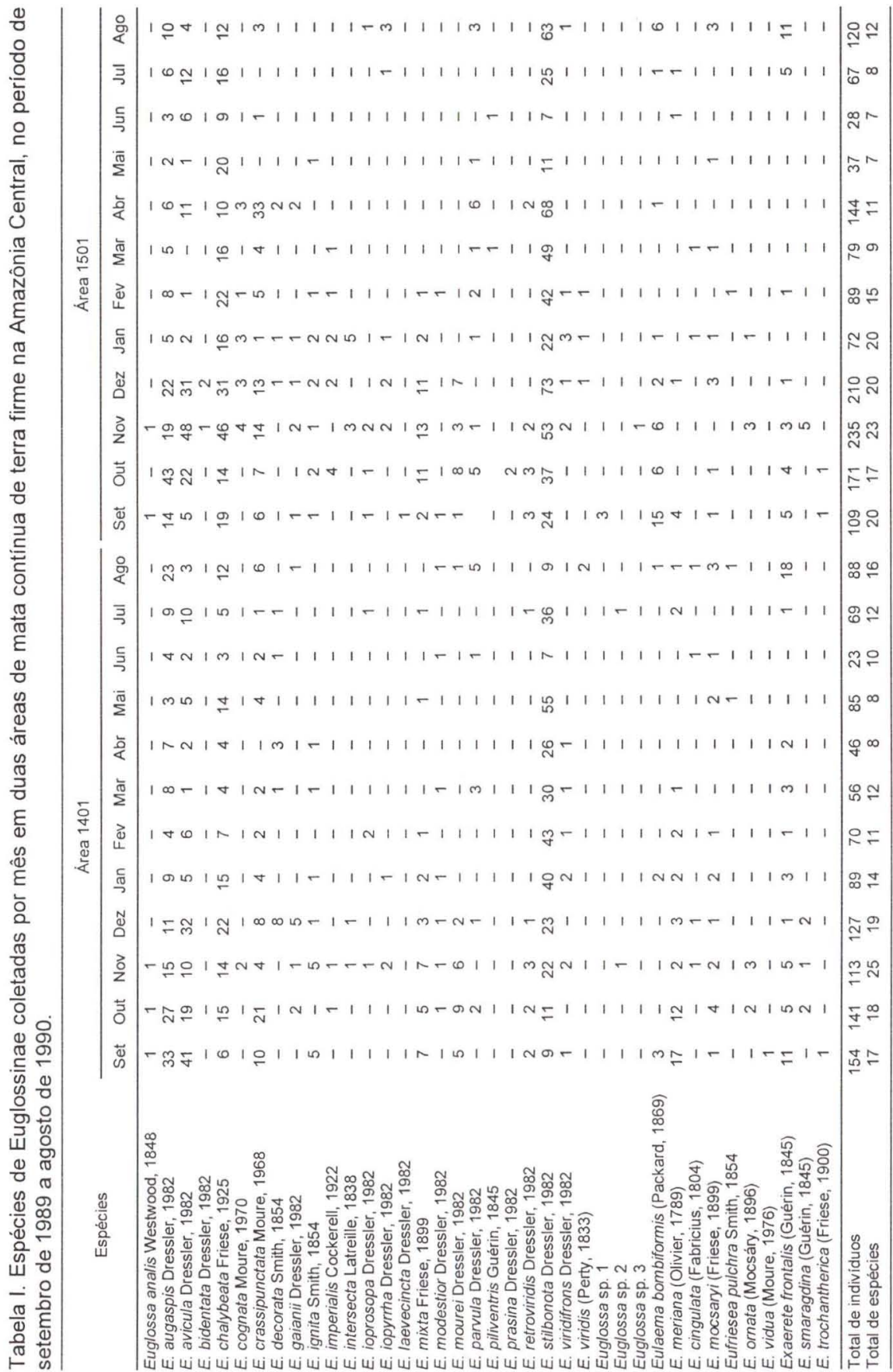

Revta bras. Zool. 16 (1): 83 - 90, 1999 
O horário de atividade foi observado em 8 ocasiões durante o ano, e, por isso, foram coletados apenas 472 indivíduos pertencentes a 34 espécies (Tab. II). O horário de atividade da maioria das espécies e indivíduos ficou compreendido entre 9 e 16 horas, para as duas áreas, sendo que nesse período as temperaturas ficaram situadas entre $24,5^{\circ}$ e $27^{\circ} \mathrm{C}$ (Figs 3, 4). Quando as temperaturas estiveram acima ou abaixo disso, a atividade foi mínima ou inexistente. $\mathrm{Na}$ área 1401 houve um pico no número de indivíduos, juntamente com um pico no número de espécies, às 11 horas, quando a temperatura esteve próxima de $26,5^{\circ} \mathrm{C}$ (Fig. 3). Diferentemente, na área 1501, um grande número de indivíduos teve seu horário de atividades se estendendo de 9 às 15 horas, com temperaturas entre $24,5^{\circ}$ e $26^{\circ} \mathrm{C}$, sem um pico definido, ao passo que um pico no número de espécies em atividade ocorreu às 14 horas (Fig. 4). Entretanto, quando as as duas áreas foram comparadas, o horário de atividade para todas as espécies apresentou uma correlação positiva ( $\mathrm{rs}=0,931$; $\mathrm{t}=6,72 ; \mathrm{p}<0,001$, G.L.=7). Houve uma correlação positiva entre espécies e indivíduos ao longo do dia para a área 1401 ( $\mathrm{rs}=0,979 ; \mathrm{t}=12,73 ; \mathrm{p}<0,001 ; \mathrm{G} . \mathrm{L}=7)$, mas nenhuma correlação para a área 1501 ( $\mathrm{rs}=0,512 ; \mathrm{t}=1,55 ; \mathrm{p}>0,05, \mathrm{G} . \mathrm{L} .=7)$.

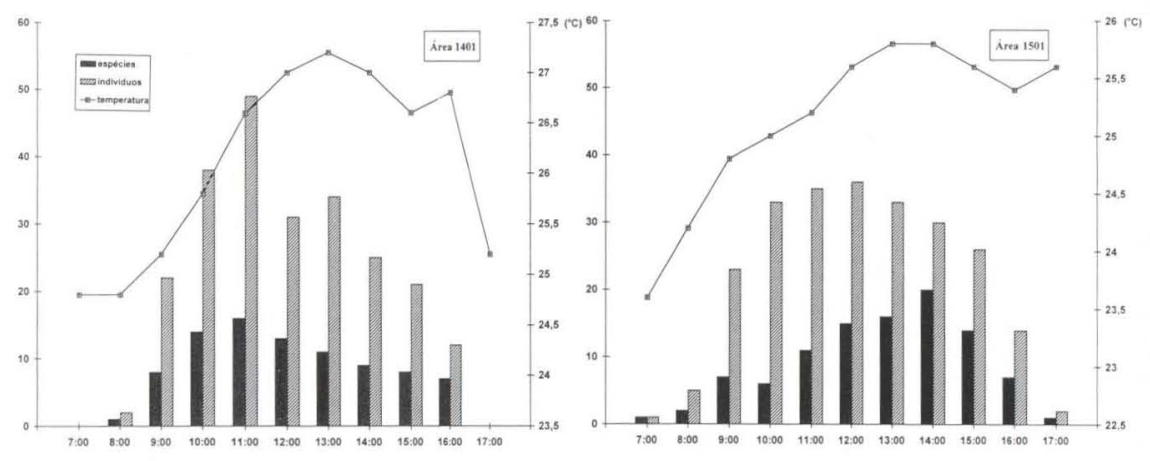

Figs 3-4. Comparaçăo do horário de atividade de Euglossinae em duas áreas de mata continua de terra firme na Amazônia Central, no período de setembro de 1989 a agosto de 1990 .

\section{DISCUSSÃO}

As espécies mais comuns apareceram predominantemente no final da estação seca e início da estação úmida. ROUBIK \& ACKERMAN (1987) em florestas tropicais secas no Panamá, PeArson \& Dressler (1985) na Amazônia Peruana, BECKER et al. (1991) em florestas tropicais úmidas de terra firme muito próximas à deste experimento e REBELO \& GARÓFALO (1991) em florestas caracterizadas como semidecíduas (LEITÃo-FiLHO 1995) e localizadas no sudeste do Brasil, também encontraram resultados semelhantes. Euglossa piliventris, a única espécie de ocorrência exclusiva na estação seca, parece ser bastante tolerante a essa estação.

Ainda não são claros os fatores que poderiam estar determinando o comportamento sazonal dos Euglossinae. JANZEN (1981) e JANZEN et al. (1982) sugerem que alguns machos de Euglossinae, em uma floresta, podem buscar substâncias odoriferas e néctar em outras florestas, ou em locais elevados nas montanhas, já que 


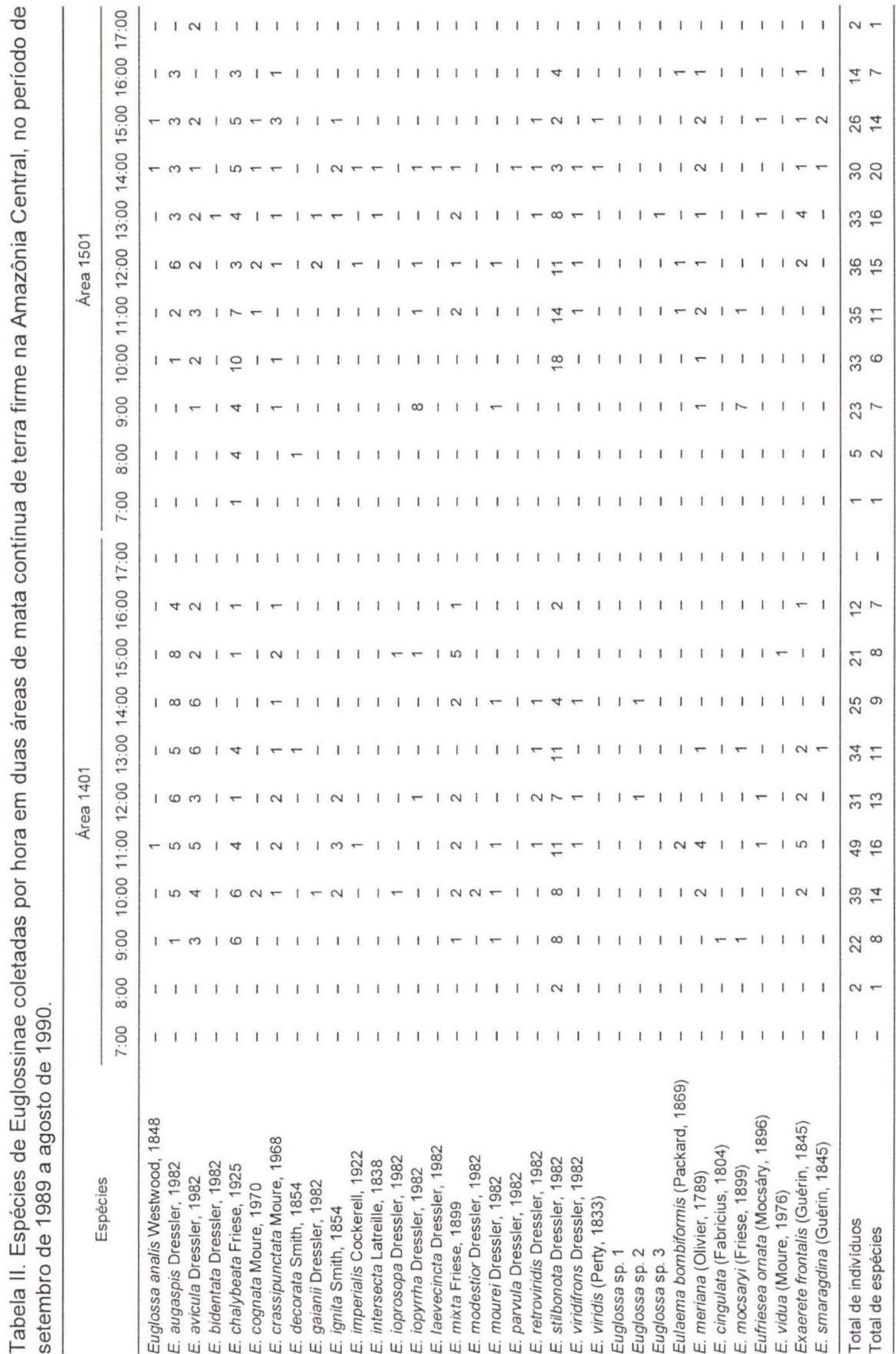


são capazes de voar a grandes distâncias e, com isso, interferir nas amostras. BECKER et al. (1991) constataram um pico na floração de Lecythidaceae, polinizada por Euglossinae, entre meados de outubro e novembro, no início da estação úmida, coincidindo com o início do pico de abundância dos Euglossinae. Para PEARSON \& DRESSLER (1985), além da fenologia da floração de certas plantas, a presença de parasitas de ninhos e o tamanho do corpo das abelhas também podem estar envolvidos. Sobre este último aspecto, MAY \& CASEY (1983) verificaram, dentre os Euglossinae, que as abelhas grandes e pubescentes como Eulaema Lepeletier, 1841 e Eufriesea Cockerell, 1908 regulam muito bem a temperatura torácica e abdominal, de acordo com a temperatura do ar, ao passo que abelhas pequenas e glabras como Euglossa Latreille, 1802 não o fazem tão eficientemente.

Muito embora, na maioria dos casos, o período de floração das orquídeas na Região Neotropical coincida com o período de atividade de seus principais polinizadores, que são os Euglossinae, parece que essas abelhas não dependem das orquídeas para obter substâncias odoriferas (ACKERMAN 1983b). Foi verificado por ZIMMERMAN et al. (1989), que a orquídea Catasetum viridiflavum Hook. tem seu pico de floração em assincronia com seu polinizador, Eulaema cingulata (Fabricius, 1804) e ACKERMAN (1983b) verificou que muitas espécies de Euglossinae coletadas na Ilha de Barro Colorado, no Panamá, não eram polinizadores das orquídeas locais. Esse autor assinalou ainda que as flutuações das populações de Euglossinae podem ser mais influenciadas por variações na disponibilidade de néctar e pólen, do que pela disponibilidade de substâncias odoríferas na natureza.

ACKERMAN (1983a), ROUBIK \& ACKERMAN (1987) e REBELO \& GARÓFALO (1991) propuseram que as flutuações sazonais na abundância das espécies de Euglossinae podem refletir atividades de nidificação e emergência de adultos. ZIMMERMAN \& MADRINAN (1988) e ACKERMAN (1989) já tinham verificado alguma variação nas preferências por substâncias odoriferas, de acordo com a idade dos machos, sendo que os primeiros autores constataram que machos jovens visitam, principalmente, substâncias odoriferas, as quais seriam necessárias para acasalamento, enquanto machos velhos visitam, principalmente, fontes de néctar. REBELO \& GARÓFAlo (1991), por sua vez, verificaram que cerca de $77 \%$ dos machos que coletavam substâncias odoríferas eram jovens.

A correlação positiva entre o número de indivíduos e de espécies ao longo do ano indica que, naqueles meses em que apareceu maior número de indivíduos, apareceu também maior número de espécies, não tendo havido, portanto, predomínio de poucas espécies com muitos indivíduos.

Dodson et al. (1969), afirmam que os Euglossinae são mais ativos na parte da manhã, e, por isso realizaram seus experimentos no período de 7 às 13 horas. Baseados nessa informação, BRAGA (1976), Powell \& Powell (1987) e BECKER et al. (1991), trabalhando na Amazônia Central, também realizaram seus experimentos em períodos semelhantes. $\mathrm{O}$ fato do presente trabalho ter sido realizado no horário de 7 às 17 horas nos permitiu verificar que o período de atividade diária dos Euglossinae é mais longo do que afirmavam esses autores.

A correlação negativa entre o número de espécies e o número de indivíduos na área 1501 , indica a predominância de poucas espécies com muitos indívíduos na 
amostra. Verificou-se, por exemplo, que ao mesmo tempo em que o número de indivíduos, ao longo do dia, aumentou até às 12 horas e depois diminuiu, o número de espécies aumentou e atingiu seu máximo por volta das 14 horas (Fig. 4). Ao que parece, tal fato pode ser atribuído a fatores climáticos ocasionais, que ocorreram especificamente nesta área, beneficiando essas poucas espécies. Provavelmente, o principal deles tenha sido a temperatura, pois foi verificado que tanto espécies quanto indivíduos estiveram mais ativos quando a temperatura do ar esteve entre 24,5 e $26,5^{\circ} \mathrm{C}$, nesta área. BRAGA (1976) assinalou que a luminosidade e a umidade relativa também devem estar envolvidas na determinação do horário de atividades. No entanto, esses fatores variam muito pouco no interior de florestas contínuas de terra firme durante o dia.

Fatores bióticos, como a disponibilidade de substâncias odoriferas, também podem estar envolvidos na determinação dos horários de atividades. SOARES et al. (1989) verificaram que machos de Euglossa mandibularis Friese, 1899 iniciavam suas visitas às flores de alguns indivíduos de Cyphomandra calycina Sendt (Solanaceae) às 5:30 horas, coletando substâncias odoríferas nos conectivos das flores abertas no dia anterior e que a partir das 9:00 horas, com a antese de novas flores, muitos indivíduos de E. mandibularis visitavam estas flores.

Ao que tudo indica, não apenas um, como sugerem alguns autores, mas vários fatores bióticos e abióticos podem estar influenciando o comportamento sazonal e diário das abelhas Euglossinae.

AGRADECIMENTOS. Ao Projeto Dinâmica Biológica de Fragmentos Florestais (PDBFF/INPA-SI) pelo auxílio financeiro e apoio nas atividades de campo. A Elder Morato, Fernando Zanella e Maria Cristina Gaglianone pelas sugestões.

\section{REFERÊNCIAS BIBLIOGRÁFICAS}

ACKERMAN, J.D. 1983a. Diversity and seasonality of male euglossine bees (Hymenoptera, Apidae) in Central Panama. Ecology 64 (2): 274-283.

. 1983b. Specificity and mutual dependency of the orchid-euglossine bee interaction. Biol. Jour. Linnean Soc. 20: 301-314.

1989. Geographic and seasonal variation in fragrance choices and preferences of male euglossine bees. Biotropica 21 (4): 340-347.

BeCKer, P.; J.S. Moure \& F.J.A. PERALTA. 1991. More about euglossine bees in amazonian forest fragments. Biotropica 23 (4b): 586-591.

BRAGA, P.I.S. 1976. Atração de abelhas polinizadoras de Orchidaceae com auxílio de iscas-odores na campina, campinarana e floresta tropical úmida da região de Manaus. Ciência e Cultura 28 (7): 767-773.

Dodson, C.H.; R.L. Dressler; H.G. Hills; R.M. Adams \& N.H. Williams. 1969. Biologically active compounds in orchid fragrances. Science 164 (13): 1243-1249.

JANZEN, D.H. 1981. Reduction in euglossine bees species richness on Isla del Cano,

a Costa Rican offshore island. Biotropica 13 (3): 238-240.

Janzen, D.H.; P.J. DE VRIES; M.L. Higgins \& L.S. KimSEY. 1982. Seasonal and 
site variation in Costa Rican euglossine bees at chemical baits in lowland deciduous forest and evergreen forests. Ecology 63 (1): 66-74.

LEITÃo-FiLHO, H. DE F. 1995. A vegetação da reserva de Santa Genebra, p.19-29. In: P.C. Morellato \& H. DE F. LEITÃo-FILHO (Eds). Ecologia e preservação de uma floresta tropical urbana: Reserva de Santa Genebra. Campinas, Editora da Unicamp, 136p.

MAY, M.L. \& T.M. CASEY. 1983. Thermoregulation and heat exchange in euglossine bees. Physiol. Zool. 56 (4): 541-551.

OliveirA, M.L. \& L.A.O. CAMPos. 1995. Abundância, riqueza e diversidade de abelhas Euglossinae (Hymenoptera, Apidae) em florestas contínuas de terra firme na Amazônia Central, Brasil. Revta bras. Zool. 12 (3): 547-556.

PEARson, D.L. \& R.L. Dressler. 1985. Two-year study of male orchid bee (Hymenoptera, Apidae, Euglossini) attraction to chemical baits in lowland south-eastern Peru. Jour. Trop. Ecol. 1: 37-54.

Powell, A.H. \& G.N.V. PowelL. 1987. Population dynamics of male euglossine bees in amazonian forest fragments. Biotropica 19 (2): 176-179.

REBELO, J.M.M. \& C.A. GARÓFALO. 1991. Diversidade e sazonalidade de machos de Euglossini (Hymenoptera, Apidae) e preferência por iscas odores em um fragmento de floresta no sudeste do Brasil. Rev. Brasil. Biol. 51 (4): 787-799.

RIBEIRO, M.N.G. \& J. ADIS. 1984. Local rainfall variability-a potential bias for bioecological studies in the Central Amazon. Acta Amazonica 14 (1- 2): 159-174.

ROUBIK, D.W. \& J.D. ACKERMAN. 1987. Long-term ecology of euglossine orchidbees (Apidae, Euglossini) in Panama. Oecologia 73: 321-333.

SOARES, A.A.; L.A.O. CAMPOS; M.F. VIEIRA \& G.A.R. MELO. 1989. Relações entre Euglossa (Euglossela) mandibularis Friese, 1899 (Hymenoptera, Apidae, Euglossini) e Cyphomandra calycina (Solanaceae). Ciência e Cultura 41 (9): 903-905.

ZIMMERMAN, J.K.; D.W. ROUBIK \& J.D. ACKERMAN. 1989. Asynchronous phenologies of a neotropical orchid and its euglossine bee pollinator. Ecology 70 (4): 1192-1195.

Zimmerman, J.K. \& S.R. MAdrinan. 1988. Age structure of male Euglossa imperialis (Hymenoptera, Apidae, Euglossini) at nectar and chemical sources in Panama. Jour. Trop. Ecol. 4: 303-306.

Recebido em 19.IX.1997; aceito em 25.I.1999. 